\title{
An Approach to the Problem of Closure in Non-Linear Stochastic Mechanics*
}

\author{
NGUYEN DONG ANH and WERNER SCHIEHLEN \\ University of Stuttgart, Institute B of Mechanics, Pfaffenwaldring 9, 70550 Stuttgart, Germany
}

(Received: 23 October 1991; accepted in revised form 24 November 1992)

\begin{abstract}
An approach combining the method of moment equations and the statistical linearization technique is proposed for analysis of the response of non-linear mechanical systems to random excitation. The adaptive statistical linearization procedure is developed for obtaining a more accurate mean square of responses. For these, a Duffing oscillator and an oscillator with cubic non-linear damping subject to white noise excitation are considered. It is shown that the adaptive statistical linearization proposed yields good accurate results for both weak and strong non-linear stochastic systems.
\end{abstract}

Sommario. Si propone un procedimento per l'analisi della risposta di sistemi meccanici non lineari ad eccitazioni stocastiche, che combina il metodo delle equazioni dei momenti e la tecnica di linearizzazione statistica. Si sviluppa un procedimento di linearizzazione statistica adattativa, che permette di ottenere più accurati valori medi quadratici della risposta. Per illustrazione, si prende in esame un oscillatore di Duffing e un oscillatore con smorzamento cubico, soggetti a un'eccitazione tipo "rumore bianco"; e si mostra che la proposta linearizzazione statistica adattativa fornisce buoni risultati per sistemi sia debolmente che fortemente non lineari.

Key words: Stationary processes, statistical linearization, Duffing oscillator, random vibration, stochastic mechanics.

\section{Introduction}

Interest in the investigation of random phenomena has increased considerably over the recent years, due to various problems encountered in engineering applications. It is well known that all real engineering systems are, more or less, non-linear and for those systems an exact solution exists only for very few special cases of limited practical value. Consequently, it is a logical approach to develop approximate methods for the analysis of non-linear stochastic systems. As mentioned by Wu and Lin [1], an approximate technique should meet three criteria: accuracy, simplicity and versatility. The general idea of the approximate methods available is to replace equations that are non-linear by equations that are easier to solve, using a certain sense of equivalence. If the latter equations are linear one is dealing with statistical linearization. Furthermore, if the coefficients of the linearized equations are evaluated by an assumption about the Gaussianity of the system response the statistical linearization used is known as Gaussian or normal. It is shown by many authors (see e.g. [2]), that this kind of linearization is the simplest and most useful tool for analysis of non-linear statistical problems. However, this technique is limited by two restrictions. First, the accuracy of the Gaussian statistical linearization decreases as the non-linearity increases. Second, it only yields estimates of the first and second moments of the response.

An alternative approach to non-linear problems is the method of moment equations, which can give estimates of all the moments of the response. However, the difficulty with this approach is that one is led to the well-known problem of closure, (see [3], [4]). There is a method of combining these two approaches, namely the first and the second moments are

\footnotetext{
- Presented at the First European Solid Mechanics Conference, September 9-13, 1991, Munich, Germany
} 


\section{0}

\section{Nguyen Dong Anh and Werner Schiehlen}

determined by statistical linearization, and the other higher moments are found from the moment equations. In this paper, such a 'middle' approach will be presented.

\section{Problem of Closure}

Consider, as an example, the Duffing oscillator

$$
\ddot{x}+2 h \dot{x}+\omega_{0}^{2} x+\gamma x^{3}=\sigma \xi(t)
$$

with the displacement variable $x(t)$. In this equation, a dot denotes time differentiation, $h$ and $\omega_{0}$ are the damping constant and the natural frequency, respectively. The constant $\gamma$ quantifies the non-linearity of the stiffness. The excitation $\xi(t)$ is a zero mean Gaussian white noise with autocorrelation

$$
R_{\xi}(\tau)=\langle\xi(t) \xi(t+\tau)\rangle=\delta(\tau) .
$$

where \langle\rangle denotes the expectation, and $\delta(\tau)$ is Dirac's delta function. It is well known that, for stationary responses, the velocity $\dot{x}(t)$ is a Gaussian random variable, which is independent of the displacement $x(t)$. Furthermore, the second moment of velocity is

$$
\left\langle\dot{x}^{2}\right\rangle=\sigma^{2} / 4 h \text {. }
$$

Since the velocity $\dot{x}(t)$ is a Gaussian random variable, all its odd moments vanish, while the even ones are

$$
\left\langle\dot{x}^{2 n}\right\rangle=(2 n-1) ! !\left\langle\dot{x}^{2}\right\rangle^{n}
$$

where

$$
(2 n-1) ! !=1 \cdot 3 \cdot 5 \cdot .(2 n-1) .
$$

Dealing with the displacement statistics, the following moment equation can be obtained using the Fokker-Planck equation approach

$$
\left\langle\frac{\mathrm{d} \psi(x)}{\mathrm{d} x}\right\rangle=\frac{4 h}{\sigma^{2}}\left(\omega_{0}^{2}\langle\psi(x) x\rangle+\gamma\left\langle\psi(x) x^{3}\right\rangle\right)
$$

where $\psi(x)$ is an arbitrary function with continuous derivative. Assuming firstly $\psi(x)=$ $x^{2 k+1}, k=0,1,2, \ldots$, in (5), one obtains the even moments of the displacement $\left\langle x^{2 k}\right\rangle$ as

$$
\begin{aligned}
& 1=\frac{4 h}{\sigma^{2}}\left(\omega_{0}^{2}\left\langle x^{2}\right\rangle+\gamma\left\langle x^{4}\right\rangle\right), \\
& 3\left\langle x^{2}\right\rangle=\frac{4 h}{\sigma^{2}}\left(\omega_{0}^{2}\left\langle x^{4}\right\rangle+\gamma\left\langle x^{6}\right\rangle\right), \ldots, \\
& (2 k+1)\left\langle x^{2 k}\right\rangle=\frac{4 h}{\sigma^{2}}\left(\omega_{0}^{2}\left\langle x^{2 k+2}\right\rangle+\gamma\left\langle x^{2 k+4}\right\rangle\right) .
\end{aligned}
$$

Assuming secondly $\psi(x)=x^{2 k}, k=0,1,2, \ldots$, in (5) it is found that all odd moments of the displacement $\left\langle x^{2 k+1}\right\rangle$ vanish. The system of even moment equations (6) is an infinite hierachy in the sense that the equation for the second moment $\left\langle x^{2}\right\rangle$ contains the fourth moment $\left\langle x^{4}\right\rangle$ and the equation for $\left\langle x^{4}\right\rangle$ contains the sixth moment $\left\langle x^{6}\right\rangle$, etc. Thus one is led to the well known 'problem of closure' which is how to truncate the system of moment equations (6). In 
the recent years some closure schemes have been presented; for example, the Gaussian closure, the equivalent linearization [2], the non-Gaussian closure using a truncated Gram-Charlier expansion [4,5,6], the cumulant-neglect closure [1] using the technique of an equivalent nonlinear equation $[7,8]$. Before selecting a closure scheme, it should be noted that, if the lower even moment of the displacement, i.e. the second moment $\left\langle x^{2}\right\rangle$, is approximately known, from the system (6), all the higher even moments will be approximately found, respectively,

$$
\begin{aligned}
& \gamma\left\langle x^{4}\right\rangle=\frac{\sigma^{2}}{4 h}-\omega_{0}^{2}\left\langle x^{2}\right\rangle, \\
& \gamma\left\langle x^{6}\right\rangle=\frac{3 \sigma^{2}}{4 h}\left\langle x^{2}\right\rangle-\omega_{0}^{2}\left\langle x^{4}\right\rangle, \ldots, \\
& \gamma\left\langle x^{2 k+4}\right\rangle=\frac{(2 k+1) \sigma^{2}}{4 h}\left\langle x^{2 k}\right\rangle-\omega_{0}^{2}\left\langle x^{2 k+2}\right\rangle .
\end{aligned}
$$

Therefore, the next question is reduced to the determination of the second moment $\left\langle x^{2}\right\rangle$, which can be found by using statistical linearization.

\section{Gaussian Statistical Linearization (GSL)}

To describe the basic idea of statistical linearization we consider the non-linear stochastic equation

$$
\ddot{x}+2 h \dot{x}+\omega_{0}^{2} x+\gamma_{n} x^{2 n+1}=f(t)
$$

where $h, \omega_{o}, \gamma_{n}$ are positive constants, $n=1,2,3, \ldots$, and the excitation $f(t)$ is a zero mean Gaussian stationary process with the correlation function given by

$$
R_{f}(\tau)=\langle(f(t) f(t+\tau))\rangle .
$$

Following the statistical linearization method, the non-linear Equation (8) is replaced by a linear equation

$$
\ddot{x}+2 h \dot{x}+\omega_{e}^{2} x=f(t)
$$

where

$$
\omega_{e}^{2}=\omega_{0}^{2}+\gamma_{n} \lambda_{n} .
$$

To find the linearized coefficient $\gamma_{n} \lambda_{n}$ it is necessary to minimize the expected value of the difference between ( 8 ) and (10)

$$
\epsilon=\gamma_{n}\left(x^{2 n+1}-\lambda_{n} x\right)
$$

in a least mean square sense. Thus, from

$$
\frac{d}{d \lambda_{n}}\left\langle\epsilon^{2}\right\rangle=0
$$

it follows

$$
\lambda_{n}=\frac{\left\langle x^{2 n+2}\right\rangle}{\left\langle x^{2}\right\rangle} .
$$




\section{2}

\section{Nguyen Dong Anh and Werner Schiehlen}

Introducing the quantity

$$
K_{n}=\frac{\left\langle x^{2 n+2}\right\rangle}{\left\langle x^{2}\right\rangle^{n+1}},
$$

Equation (14) can be rewritten as

$$
\lambda_{n}=K_{n}\left\langle x^{2}\right\rangle^{n}
$$

and then, from (11), one gets

$$
\omega_{e}^{2}=\omega_{0}^{2}+\gamma_{n} K_{n}\left\langle x^{2}\right\rangle^{n} .
$$

In the general case, the expectations indicated in (14) and (15) are evaluated for the statistics of the original non-linear system (8), and therefore, the value of $K_{n}$ depends upon the system parameters

$$
K_{n}=K_{n}\left(h, \omega_{0}^{2}, \gamma_{n}, \sigma\right)
$$

where $\sigma$ is the intensity of the random excitation $f(t)$. For Gaussian statistical linearization (GSL) the response process $x(t)$ is assumed to be Gaussian, thus one gets

$$
K_{n}=(2 n+1) ! !
$$

using the definition from (4) again. Substituting (19) into (17), the Gaussian expression for the equivalent frequency $\omega_{e}^{2}$ is obtained:

$$
\omega_{e}^{2}=\omega_{0}^{2}+\gamma_{n}(2 n+1) ! !\left\langle x^{2}\right\rangle^{n} .
$$

Hence, the GSL method replaces the function (18) by the constant value $(2 n+1)$ ! !, assuming the Gaussianity of the response. In this sense, the GSL is insufficient and that is why, generally speaking, it can give an accurate quantitative result only for weak non-linear systems $[9,10]$.

\section{Adaptive Statistical Linearization (ASL)}

Statistical linearization, in which the value $K_{n}$ of (18) is considered to be a function of the system parameters, will be called here adaptive. Evidently, ASL should yield more accurate results than GSL. However, the exact evaluation of the function $K_{n}\left(h, \omega_{0}^{2}, \gamma_{n}, \sigma\right)$ requires a knowledge of the unknown probability density function of the response process $x(t)$. A question then arises: how can the function $K_{n}\left(h, \omega_{0}^{2}, \gamma_{n}, \sigma\right)$ be constructed? First of all, it is seen from (8) that the degree of system non-linearity can be defined as the ratio $\gamma_{n} / \omega_{0}^{2}$. Then the function $K_{n}$ can be expanded in the form of the powers $\gamma_{n} / \omega_{0}^{2}$ :

$$
K_{n}=\frac{\left\langle x^{2 n+2}\right\rangle}{\left\langle x^{2}\right\rangle^{n+1}}=\frac{\left\langle x_{0}^{2 n+2}\right\rangle\left[1+A_{1 n} \frac{\gamma_{n}}{\omega_{0}^{2}}+A_{2 n}\left(\frac{\gamma_{n}}{\omega_{0}^{2}}\right)^{2}+\cdots\right]}{\left\langle x_{0}^{2}\right\rangle^{n+1}\left[1+B_{1 n} \frac{\gamma_{n}}{\omega_{0}^{2}}+B_{2 n}\left(\frac{\gamma_{n}}{\omega_{0}^{2}}\right)^{2}+\cdots\right]}
$$

where $\left\langle x_{0}^{2 n+2}\right\rangle,\left\langle x_{0}^{2}\right\rangle$ denote the moments of response of the corresponding linear system ' $\left.\gamma_{n}=0\right)$, i.e.

$$
\ddot{x}_{0}+2 h \dot{x}_{0}+\omega_{0}^{2} x_{0}=f(t)
$$




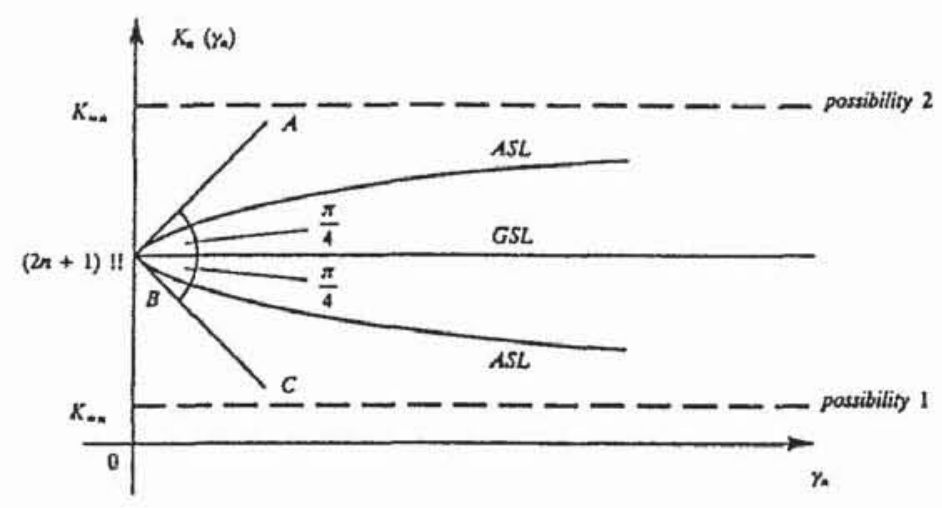

Fig. 1. Function $K_{n}$ over nonlinearity $\gamma_{n}$.

and $A_{k n}, B_{k n}, k=1,2, \ldots$, are the coefficients depending on $h, \sigma$. Hence, one reads immediately

$$
\frac{\left\langle x_{0}^{2 n+2}\right\rangle}{\left\langle x_{0}^{2}\right\rangle^{n+1}}=(2 n+1) ! ! \text {. }
$$

Substituting (23) into (21) and neglecting the terms of the powers of $\left(\gamma_{n} / \omega_{0}^{2}\right)$ higher then 1 , it remains

$$
K_{n}=\frac{\left\langle x^{2 n+2}\right\rangle}{\left\langle x^{2}\right\rangle^{n+1}}=(2 n+1) ! ! \frac{\omega_{0}^{2}+A_{1 n} \gamma_{n}}{\omega_{0}^{2}+B_{1 n} \gamma_{n}} .
$$

Denote

$$
K_{\infty n}(h, \sigma)=\lim _{\gamma_{n} / \omega_{0}^{2} \rightarrow \infty} K_{n}=\lim _{\gamma_{n} / \omega_{0}^{2} \rightarrow \infty} \frac{\left\langle x^{2 n+2}\right\rangle}{\left\langle x^{2}\right\rangle^{n+1}}
$$

Substituting (24) into (25) gives

$$
(2 n+1) ! ! \frac{A_{1 n}}{B_{1 n}}=K_{\infty n} .
$$

Eliminating $A_{1 n}$ from (24) using (26) yields

$$
K_{n}=\frac{(2 n+1) ! ! \omega_{0}^{2}+B_{1 n} K_{\infty n} \gamma_{n}}{\omega_{0}^{2}+B_{1 n} \gamma_{n}} .
$$

The value $B_{1 n}$ indicates the velocity of departure of the value $K_{n}$ from the value $(2 n+1) !$ ! Indeed one gets

$$
\frac{\partial K_{n}}{\partial \gamma_{n}}=\frac{B_{1 n}\left(K_{\infty n}-(2 n+1) ! !\right) \omega_{0}^{2}}{\left(\omega_{0}^{2}+B_{1 n} \gamma_{n}\right)^{2}} .
$$

In principle, the value $B_{1 n}$ could be estimated using the perturbation method [11]; however, it might be complicated. Hence, for practical applications the following consideration is proposed. As shown in Figure 1, in the case of GSL the curve $K_{n}\left(\gamma_{n}\right)$ becomes a horizontal line through B. From (19) one gets $\partial K_{n} / \partial \gamma_{n}=0$. 


\section{4}

On the other hand, as has been observed by many authors, the response process of the system $(8)$ is closed to the Gaussian one for very small non-linearities $\gamma_{n} \approx 0$. So for ASL the following requirement is met, $\partial K_{n} / \partial \gamma_{n} \mid \gamma_{n}=0 \approx 0$. This condition together with (28) results in

$$
B_{1 n}\left(K_{\infty n}-(2 n+1) ! !\right) \approx 0 .
$$

For the further analysis it is assumed

$$
-1=\tan \left(-\frac{\pi}{4}\right)<B_{1 n}\left(K_{\infty n}-(2 n+1) ! !\right)<\tan \left(\frac{\pi}{4}\right)=1 .
$$

The inequalities (30) assures that the curve $K_{n}\left(\gamma_{n}\right)$ is kept in triangle $\mathrm{ABC}$ when $\gamma_{n} \approx 0$ (Figure 1). Thus, in the case of ASL, $B_{1 n}$ is given by any value satisfying (30). It is seen from (27), when the non-linearity is small $\gamma_{n} \approx 0$, one gets $K_{n} \approx(2 n+1)$ !! ! Thus in this case ASL involves GSL. In the general case, the adaptive expression for the equivalent frequency $\omega_{e}^{2}$ depends on $B_{1 n}$ and can be obtained, substituting (27) into (17),

$$
\omega_{e}^{2}=\omega_{0}^{2}+\gamma_{n} \frac{(2 n+1) ! ! \omega_{0}^{2}+B_{1 n} K_{\infty n} \gamma_{n}}{\omega_{0}^{2}+B_{1 n} \gamma_{n}}\left\langle x^{2}\right\rangle^{n} .
$$

\section{Approximate Estimate for $K_{\infty n}$}

As seen from (31) and (30), a knowledge of the value $K_{\infty n}$ leads to a suitable equivalent frequency $\omega_{e}^{2}$. To find, approximately, the value $K_{\infty n}$ we consider the two limited cases of (8). First, choosing $\gamma_{n}=0$ in (8) yields to the linear equation (22) and then one gets

$$
K_{\infty n}^{0}=(2 n+1) ! ! \text {. }
$$

Suppose then (8) has very strong nonlinearity, $\gamma_{n} \gg 1+2 h+\omega_{n}^{2}$, then the linear terms in (8) can be neglected. As a result, one gets

$$
\gamma_{n} x^{2 n+1}=f(t) \text {. }
$$

Equation (32) represents a transformation of the random variables $x(t)$ and $f(t)$. Therefore, the statistic of the variable $x(t)$ can be calculated easily (see Appendix). Then, it follows

$$
K_{\infty n}^{1}=\frac{\left\langle x^{2 n+2}\right\rangle}{\left\langle x^{2}\right\rangle^{n+1}}=\sqrt{\pi^{n}} \frac{\Gamma((4 n+3) /(4 n+2))}{\Gamma^{n+1}((2 n+3) /(4 n+2))} .
$$

Consider now (8) for the general case with a linear and a non-linear part. The following expression for $K_{\infty n}$ could be proposed

$$
K_{\infty n}=d_{n}\left((2 n+1) ! !+\sqrt{\pi^{n}} \frac{\Gamma((4 n+3) /(4 n+2))}{\Gamma^{n+1}((2 n+3) /(4 n+2))}\right)
$$

where $d_{n}$ is a constant coefficient. In the linear case, $n=0$, the coefficient is $d_{0}=\frac{1}{2}$. For the non-linear case, $n>0$, the coefficient $d_{n}$ will also be a function of the damping constant $h$ and the excitation intensity $\sigma$ [see (25) and (34)] $d_{n}=d_{n}(h, \sigma)$, and needs further investigations. In this paper, however, the value $d_{n}=\frac{1}{2}$ is taken. So one has

$$
K_{\infty n}=\frac{1}{2}\left((2 n+1) ! !+\sqrt{\pi^{n}} \frac{\Gamma((4 n+3) /(4 n+2))}{\Gamma^{n+1}((2 n+3) /(4 n+2))}\right) .
$$

Substituting (35) into (31) shows that the equivalent frequency $\omega_{e}^{2}$ is readily determined. 


\section{Oscillator with Non-Linear Stiffness}

The result just obtained can be easily extended to a single degree of freedom system with linear damping and non-linear spring. The equation of motion of such a system is given by

$$
\ddot{x}+2 h \dot{x}+\omega_{0}^{2} x+\sum_{n=1}^{N} \gamma_{n} x^{2 n+1}=f(t) .
$$

Here $\gamma_{n}$ are positive constants, $f(t)$ is a random excitation as in (8). Using the ASL method, the non-linear equation (36) is replaced by a linear equation

$$
\ddot{x}+2 h \dot{x}+\omega_{e}^{2} x=f(t)
$$

where according to (31) it yields

$$
\omega_{e}^{2}=\omega_{0}^{2}+\sum_{n=1}^{N} \gamma_{n} \frac{(2 n+1) ! ! \omega_{0}^{2}+B_{1 n} K_{\infty n} \gamma_{n}}{\omega_{0}^{2}+B_{1 n} \gamma_{n}}\left\langle x^{2}\right\rangle^{n}
$$

The values of $K_{\infty n}$ are given in (35) and $B_{1 n}$ are chosen from (30). The next step is to evaluate $\left\langle x^{2}\right\rangle$ using the linearized equation (37),

$$
\left\langle x^{2}\right\rangle=\int_{-\infty}^{+\infty} \frac{S_{f}(\omega) d \omega}{\left(\omega_{e}^{2}-\omega^{2}\right)^{2}+4 h^{2} \omega^{2}}
$$

where $S_{f}(\omega)$ is the spectral density of the random excitation $f(t)$. Equations (38) and (39) yield a relationship for the mean square response $\left\langle x^{2}\right\rangle$. Further, $\left\langle\dot{x}^{2}\right\rangle$ can be found from

$$
\left\langle\dot{x}^{2}\right\rangle=\int_{-\infty}^{+\infty} \frac{S_{f}(\omega) \omega^{2} d \omega}{\left(\omega_{e}^{2}-\omega^{2}\right)^{2}+4 h^{2} \omega^{2}} .
$$

In the case of white noise excitation

$$
S_{f}(\omega)=\frac{\sigma^{2}}{2 \pi}
$$

(39) gives simply

$$
\left\langle x^{2}\right\rangle=\frac{\sigma^{2}}{4 h \omega_{e}^{2}} .
$$

Substituting (42) into (38) yields the following algebraic equation

$$
\left\langle x^{2}\right\rangle\left[\omega_{0}^{2}+\sum_{n=1}^{N} \gamma_{n} \frac{(2 n+1) ! ! \omega_{0}^{2}+B_{1 n} K_{\infty n} \gamma_{n}}{\omega_{0}^{2}+B_{1 n} \gamma_{n}}\left\langle x^{2}\right\rangle^{n}\right]-\frac{\sigma^{2}}{4 h}=0
$$

which finally determines the mean square response $\left\langle x^{2}\right\rangle$. 


\section{6}

\section{Nguyen Dong Anh and Werner Schiehlen}

\section{Oscillator with Non-Linear Damping}

It will be shown herein how the ASL method can be used for the oscillators with non-linear damping. Consider the equation

$$
\ddot{x}+2 h \dot{x}+\sum_{n=1}^{n} \beta_{n} \dot{x}^{2 n+1}+\omega_{0}^{2} x=f(t)
$$

where $h, \beta_{n}, \omega_{0}$ are positive constants, and the random excitation, $f(t)$, as in (8). Following the ASL method, the non-linear equation is replaced by a linear equation

$$
\ddot{x}+h_{e} \dot{x}+\omega_{0}^{2} x=f(t) .
$$

The equivalent damping coefficient $h_{e}$ is defined, as usual, as

$$
h_{e}=2 h+\sum_{n=1}^{N} \beta_{n} H_{n}\left\langle\dot{x}^{2}\right\rangle^{n}
$$

where

$$
H_{n}=\frac{\left\langle\dot{x}^{2 n+2}\right\rangle}{\left\langle\dot{x}^{2}\right\rangle^{n+1}} .
$$

The procedure for obtaining $H_{n}\left(h, \omega_{0}^{2}, \beta_{n}, \sigma\right)$ follows that for $K_{n}\left(h, \omega_{0}^{2}, \gamma_{n}, \sigma\right)$ in Sections 4 and 5 , except that the non-linearity degree is indicated by the ratio

$$
\frac{\beta_{n}}{2 h} \text {. }
$$

Thus, instead of the value $\gamma_{n} / \omega_{0}^{2}$ one can use $\beta_{n} / 2 h$ and for $H_{n}$ one obtains an expression corresponding to (27),

$$
H_{n}=\frac{(2 n+1) ! ! 2 h+B_{1 n} K_{\infty n} \beta_{n}}{2 h+B_{1 n} \beta_{n}},
$$

where $K_{\infty n}$ is given by (35) and $B_{1 n}$ is chosen from (30).

Substituting (48) into (46) yields the following expression for the equivalent damping coefficient

$$
h_{e}=2 h+\sum_{n=1}^{N} \beta_{n} \frac{(2 n+1) ! ! 2 h+B_{1 n} K_{\infty n} \beta_{n}}{2 h+B_{1 n} \beta_{n}}\left\langle\dot{x}^{2}\right\rangle^{n} .
$$

Using now the linearized equation (45) gives

$$
\left\langle\dot{x}^{2}\right\rangle=\int_{-\infty}^{+\infty} \frac{S_{f}(\omega) \omega^{2} d \omega}{\left(\omega_{0}^{2}-\omega^{2}\right)^{2}+h_{e}^{2} \omega^{2}} .
$$

Equation (50), (49), (35) and condition (30) form a relationship for determining the velocity mean square $\left\langle\dot{x}^{2}\right\rangle$. The mean square of displacement $\left\langle x^{2}\right\rangle$ can be found later by the formula

$$
\left\langle x^{2}\right\rangle=\int_{-\infty}^{+\infty} \frac{S_{f}(\omega) d \omega}{\left(\omega_{0}^{2}-\omega^{2}\right)^{2}+h_{e}^{2} \omega^{2}} .
$$


In the case of white noise excitation (41), (50) yields

$$
\left\langle\dot{x}^{2}\right\rangle=\sigma^{2} / 2 h_{e} .
$$

Substituting (49) into (52) gives the following algebraic equation

$$
\left\langle\dot{x}^{2}\right\rangle\left[2 h+\sum_{n=1}^{N} \beta_{n} \frac{(2 n+1) ! ! 2 h+B_{1 n} K_{\infty n} \beta_{n}}{2 h+B_{1 n} \beta_{n}}\left\langle\dot{x}^{2}\right\rangle^{n}\right]-\frac{\sigma^{2}}{2}=0
$$

where the values $K_{\infty n}$ are given by (35) and $B_{1 n}$ are chosen from (30). Thus, the mean square velocity response $\left\langle\dot{x}^{2}\right\rangle$ is available from (53).

\section{Duffing Oscillator}

As an illustration the application of the ASL method will now be given for the Duffing oscillator and, then, for an oscillator with cubic non-linear damping. Firstly, consider the Duffing oscillator

$$
\ddot{x}+2 h \dot{x}+\omega_{0}^{2}+\gamma x^{3}=f(t)
$$

where the symbols have the same meanings as in (1); however, the excitation $f(t)$ is not restricted to white noise. By setting $n=1$ in (35) one has

$$
K_{\infty 1}=\frac{1}{2}\left[3+\sqrt{\pi} \frac{\Gamma(7 / 6)}{\Gamma^{2}(5 / 6)}\right]=2.147
$$

Substituting (55) into (30) and noting $n=1$, one gets

$$
-1<-B_{11} 0.853<1 .
$$

Thus, $B_{11}$ can be taken as

$$
B_{11}=1 \text {. }
$$

Substituting (55) into (31), and noting (56), yields the following expression for the equivalent frequency of the Duffing oscillator

$$
\omega_{e}^{2}=\omega_{0}^{2}+\gamma \frac{3 \omega_{0}^{2}+2.147 \gamma}{\omega_{0}^{2}+\gamma}\left\langle x^{2}\right\rangle .
$$

Thus, there are two equations (39), (57) for two unknowns $\omega_{e}^{2}$, and $\left\langle x^{2}\right\rangle$. A detailed investigation of this system will be given later. On the other hand, a probability density function for the response can be found from the calculated moments. It is well known that the estimation of the system's reliability is particularly sensitive to the character of the extreme 'tails' of the probability distribution of the response. From this point of view, in contrast to GSL, the ASL method can lead to a prediction concerning the influence of non-linearity on the distribution of the response. Thus, it can be used for the problems concerning system reliability analysis. To illustrate the approach proposed, consider the expansion of the probability density function $[2,12]$

$$
W(x)=\left[1+\sum_{K=2}^{\infty} \frac{1}{(2 K) !} \frac{b_{2 K}}{\sigma_{x}^{2 K}} H_{2 k}\left(\frac{x}{\sigma_{x}}\right)\right] W_{G}(x)
$$




\section{Nguyen Dong Anh and Werner Schiehlen}

where $W_{G}(x)$ is the Gaussian density function

$$
W_{G}(x)=\frac{1}{\sqrt{2 \pi} \sigma_{x}} \exp \left[-\frac{x^{2}}{2 \sigma_{x}^{2}}\right],
$$

$b_{2 K}$ are quasi-moments and $H_{2 K}$ are Hermite polynomials. In particular, one has

$$
b_{4}=\left\langle x^{4}\right\rangle-3\left\langle x^{2}\right\rangle^{2}, H_{4}(x)=x^{4}-6 x^{2}+3 .
$$

Using the expansion (58) up to fourth order and introducing the non-dimensional displacement $y=x / \sigma_{x}$, one gets

$$
W(y)=\frac{1}{\sqrt{2 \pi}}\left(1+\frac{b_{4}}{24\left\langle x^{2}\right\rangle^{2}}\left(y^{4}-6 y^{2}+3\right)\right) \exp \left(-\frac{y^{2}}{2}\right) .
$$

On the other hand one obtains from (57)

$$
K_{1}=\frac{\left\langle x^{4}\right\rangle}{\left\langle x^{2}\right\rangle^{2}}=\frac{3 \omega_{0}^{2}+2.147 \gamma}{\omega_{0}^{2}+\gamma} \text {. }
$$

Thus, one obtains

$$
b_{4}=\left\langle x^{4}\right\rangle-3\left\langle x^{2}\right\rangle^{2}=\frac{-0.853 \gamma\left\langle x^{2}\right\rangle^{2}}{\omega_{0}^{2}+\gamma} .
$$

Substituting (61) into (60) yields the following non-Gaussian expression for the distribution of the response

$$
W(y)=\frac{1}{\sqrt{2 \pi}}\left(1-\frac{0.853 \gamma\left(y^{4}-6 y^{2}+3\right)}{24\left(\omega_{0}^{2}+\gamma\right)}\right) \exp \left(-\frac{y^{2}}{2}\right) .
$$

As seen from (62), the distribution $W(y)$ depends on the non-linearity $\gamma$, and becomes a Gaussian distribution for $\gamma=0$. By setting $y=0$ in (62) the extreme 'tail' of the distribution is obtained as

$$
W(0)=\frac{1}{\sqrt{2 \pi}}\left(1-\frac{0.853 \gamma}{8\left(\omega_{0}^{2}+\gamma\right)}\right) .
$$

Consider now the Duffing oscillator (54) with a special random excitation.

\subsection{White Noise Excitation}

Let $f(t)$ be white noise again with

$$
\langle f(t)\rangle=0, S_{f}(w)=\sigma^{2} / 2 \pi .
$$

In this case, (39) yields

$$
\left\langle x^{2}\right\rangle=\sigma^{2} / 4 h \omega_{e}^{2} .
$$

Substituting (57) into (65) yields a quadratic equation for $\left\langle x^{2}\right\rangle$ :

$$
\left\langle x^{2}\right\rangle\left[\omega_{0}^{2}+\frac{3 \gamma \omega_{0}^{2}+2.147 \gamma^{2}}{\omega_{0}^{2}+\gamma}\left\langle x^{2}\right\rangle\right]-\frac{\sigma^{2}}{4 h}=0
$$


Table 1. Exact and approximate mean squares of displacement ([69])

\begin{tabular}{lll}
\hline$N$ & Value & Error (\%) \\
\hline$\left\langle x^{2}\right\rangle_{e}$ & $0.6760 \sqrt{d / \alpha}$ & 0 \\
$\left\langle x^{2}\right\rangle_{g}$ & $0.5774 \sqrt{d / \alpha}$ & $14.59 \%$ \\
$\left\langle x^{2}\right\rangle_{a}$ & $0.6825 \sqrt{d / \alpha}$ & $0.96 \%$ \\
\hline
\end{tabular}

which results in

$$
\left\langle x^{2}\right\rangle=\frac{\sqrt{h^{2} \omega_{0}^{4}+\sigma^{2} h \lambda}-h \omega_{0}^{2}}{2 h \lambda}
$$

where

$$
\lambda=\frac{3 \gamma \omega_{0}^{2}+2.147 \lambda^{2}}{\omega_{0}^{2}+\gamma} .
$$

The accuracy of the solution (67) will be checked by some examples. The following notations are used:

$$
\begin{aligned}
& \left\langle x^{2}\right\rangle_{e}-\text { exact solution, } \\
& \left\langle x^{2}\right\rangle_{a}-\text { solution obtained by ASL, } \\
& \left\langle x^{2}\right\rangle_{g}-\text { solution obtained by GSL. }
\end{aligned}
$$

EXAMPLE 1 [9]. Consider the Duffing oscillator with white noise excitation

$$
\begin{aligned}
& \ddot{x}+\beta \dot{x}+\alpha x^{3}=f(t), \\
& \langle f(t) f(t+\sigma)\rangle=2 d \beta \delta(\sigma) .
\end{aligned}
$$

In this case it yields

$$
h_{\iota}=\beta / 2, \omega_{0}=0, \gamma=\alpha, \sigma^{2}=2 d \beta .
$$

Thus, one gets, from (68),

$$
\lambda=2.147 \alpha
$$

and then from (67)

$$
\left\langle x^{2}\right\rangle_{a}=\frac{1}{\sqrt{2.147}} \sqrt{\frac{d}{\alpha}}=0.6825 \sqrt{\frac{d}{\alpha}} .
$$

As seen from Table I, the error of the solution obtained by ASL, $\left\langle x^{2}\right\rangle_{a}$, is $0.96 \%$ while the solution obtained by GLS, $\left\langle x^{2}\right\rangle_{g}$, has an error of $14.59 \%$. 
Table 2. Exact and approximate mean squares of displacement ([72])

\begin{tabular}{rrrrrrr}
\hline$N$ & $\epsilon$ & $\left\langle x^{2}\right\rangle_{e}$ & $\left\langle x^{2}\right\rangle_{g}$ & $\begin{array}{l}\text { Error } \\
(\%)\end{array}$ & $\left\langle x^{2}\right\rangle_{a}$ & \multicolumn{1}{l}{$\begin{array}{l}\text { Error } \\
(\%)\end{array}$} \\
\hline 1 & 0.01 & 0.9721436 & 0.9716754 & -0.48 & 0.9717508 & -0.40 \\
2 & 0.1 & 0.8175612 & 0.8053996 & -1.49 & 0.8088173 & -1.07 \\
3 & 1.0 & 0.4679198 & 0.4342586 & -7.19 & 0.4586467 & -1.98 \\
4 & 10.0 & 0.1889024 & 0.1666667 & -11.8 & 0.1907328 & 0.97 \\
5 & 100.0 & 0.0649582 & 0.0560924 & -13.6 & 0.0658330 & 1.35 \\
\hline
\end{tabular}

EXAMPLE 2 [13], [5]. Consider the Duffing oscillator

$$
\begin{aligned}
\ddot{x}+2 \eta \dot{x}+x+\epsilon x^{3} & =f(t), \\
\langle f(t) f(t+\sigma)\rangle & =4 \eta \delta(\sigma) .
\end{aligned}
$$

Comparing (72) with (54) shows

$$
h=\eta, \omega_{0}=1, \gamma=\epsilon, \sigma^{2}=4 \eta .
$$

Substituting (73) into (68), (67) yields

$$
\lambda=\frac{3 \epsilon+2.147 \epsilon^{2}}{1+\epsilon},\left\langle x^{2}\right\rangle_{a}=\frac{\sqrt{1+4 \lambda}-1}{2 \lambda} .
$$

The response mean square $\left\langle x^{2}\right\rangle$, for different values of $\epsilon$, is given in Table II, which shows, as expected, that the ASL method gives good accurate solutions for both weak and strong non-linear systems. Furthermore, while the derivation of $\left\langle x^{2}\right\rangle_{g}$ from the exact solution $\left\langle x^{2}\right\rangle_{e}$ becomes large, as the non-linearity increases, up to $13.6 \%$, the corresponding error of $\left\langle x^{2}\right\rangle_{a}$ is less than $1.36 \%$.

\section{Oscillator with Cubic Non-Linear Damping}

Consider finally an oscillator with non-linear damping, the equation of motion of which reads as

$$
\ddot{x}+2 h \dot{x}+\beta \dot{x}^{3}+\omega_{0}^{2} x=f(t) .
$$

The equivalent linear equation, corresponding to (75), takes the form

$$
\ddot{x}+h_{e} \dot{x}+\omega_{0}^{2} x=f(t)
$$

where $h_{e}$ follows from (46) and (48) as

$$
h_{e}=2 h+\beta \frac{6 h+B_{11} K_{\infty 1} \beta}{2 h+B_{11} \beta}\left\langle\dot{x}^{2}\right\rangle .
$$

Substituting (55) and (56) into (77) yields

$$
h_{e}=2 h+\beta \frac{6 h+2.147 \beta}{2 h+\beta}\left\langle\dot{x}^{2}\right\rangle \text {. }
$$


Equations (50) and (78) form a relationship to determine $\left\langle\dot{x}^{2}\right\rangle$. In the case of white noise excitation (64), (50) gives

$$
\left\langle\dot{x}^{2}\right\rangle=\sigma^{2} / 2 h_{e} \text {. }
$$

Substituting (78) into (79) yields a quadratic equation

$$
\left\langle\dot{x}^{2}\right\rangle\left[4 h+\frac{12 \beta h+4.294 \beta^{2}}{2 h+\beta}\left\langle\dot{x}^{2}\right\rangle\right]-\sigma^{2}=0
$$

with the solution

$$
\left\langle\dot{x}^{2}\right\rangle=\frac{\sqrt{4 h^{2}+\sigma^{2} \lambda}-2 h}{\lambda}
$$

where

$$
\lambda=\frac{12 \beta h+4.294 \beta^{2}}{2 h+\beta} .
$$

The mean square of the displacement $\left\langle x^{2}\right\rangle$ is determined by the formula

$$
\left\langle x^{2}\right\rangle=\left\langle\dot{x}^{2}\right\rangle \omega_{0}^{2} \text {. }
$$

EXAMPLE 3 ([2]). Consider the following oscillator with cubic non-linear damping and white noise excitation

$$
\begin{aligned}
& \ddot{x}+2 \zeta \dot{x}+2 \zeta \mu \dot{x}^{3}+x=\sqrt{4 \zeta} f(t), \\
& \langle f(t) f(t+\tau)\rangle=\delta(\tau) .
\end{aligned}
$$

As is known, exact solutions for this oscillator do not exist, even in the case of white noise excitation. Thus, the oscillator (84) has been investigated in [2] by the method of non-Gaussian closure, missing the equivalent non-linear equation (ENLE), Gaussian statistical linearization, and by the simulation. It is shown in [2], that the ENLE result, denoted as $\left\langle x^{2}\right\rangle_{E N L E}$, is nearly exact at $\zeta=0.05$ and can be used as a reference solution. This approach will be used herein for checking the accuracy of the adaptive statistical linearization solution. Thus, comparing (75) and (84) gives

$$
h=\zeta, \beta=2 \zeta \mu, \omega_{0}=1, \sigma^{2}=4 \zeta .
$$

Substituting (85) into (82), (81) and (83) yields

$$
\lambda=\frac{12 \zeta \mu+8.588 \zeta \mu^{2}}{1+\mu},
$$

and

$$
\left\langle x^{2}\right\rangle=\frac{2\left(\sqrt{\zeta^{2}+\zeta \lambda}-\zeta\right)}{\lambda} .
$$

In the case of $\zeta=0.05$, one gets

$$
\begin{aligned}
& \lambda=\frac{0.6 \mu+0.4294 \mu^{2}}{1+\mu} \\
& \left\langle x^{2}\right\rangle_{a}=\frac{2(\sqrt{0.0025+0.05 \lambda}-0.05)}{\lambda} .
\end{aligned}
$$


Table 3. Approximate mean squares of displacement ([84])

\begin{tabular}{rllllc}
\hline$\mu$ & $\left\langle x^{2}\right\rangle_{\text {ENLE }}$ & $\left\langle x^{2}\right\rangle_{g}$ & Error $(\%)$ & $\left\langle x^{2}\right\rangle_{a}$ & Error (\%) \\
\hline 1 & 0.4603 & 0.4342 & -5.67 & 0.4586 & -0.37 \\
2 & 0.3584 & 0.3333 & -7.00 & 0.3632 & 1.06 \\
3 & 0.3058 & 0.2824 & -7.65 & 0.3128 & 2.29 \\
4 & 0.2720 & 0.2500 & -8.09 & 0.2789 & 2.54 \\
5 & 0.2476 & 0.2270 & -8.43 & 0.2551 & 2.90 \\
6 & 0.2294 & 0.2096 & -8.63 & 0.2368 & 3.23 \\
7 & 0.2147 & 0.1957 & -8.85 & 0.2221 & 3.45 \\
8 & 0.2025 & 0.1844 & -8.94 & 0.2099 & 3.65 \\
9 & 0.1923 & 0.1748 & -9.10 & 0.1996 & 3.80 \\
10 & 0.1835 & 0.1667 & -9.16 & 0.1907 & 3.92 \\
\hline
\end{tabular}

The accuracy of the ASL solution, $\left\langle x^{2}\right\rangle_{a}$, given by (88), is shown in Table III for different values of $\mu$ where the solution $\left\langle x^{2}\right\rangle_{\mathrm{ENLE}}$, and $\left\langle x^{2}\right\rangle_{g}$ are taken from Table 9.3 in [2]. Obviously, the solution obtained by the ASL method, $\left\langle x^{2}\right\rangle_{a}$, is much closer to the solution $\left\langle x^{2}\right\rangle_{\mathrm{ENLE}}$, than the solution obtained by the GSL method, $\left\langle x^{2}\right\rangle_{g}$.

\section{Conclusions}

The main question inherent in statistical linearization is how the equivalent coefficients of the linearized equation are found. Gaussian statistical linearization (GSL) proposes these coefficients to be constant values, while the adaptive statistical linearization (ASL) considers those as the adaptive functions of the system parameters. In the examples investigated the ASL method yields better results for both weak and strong non-linear stochastic systems. Thus, adaptive statistical linearization seems to be a more sophisticated approach for non-linear stochastic systems.

\section{Acknowledgement}

This research was supported partly by the Alexander von Humboldt Foundation.

\section{Appendix}

Let $f(t)$ be zero-mean Gaussian process with the probability density function

$$
W(f)=(\sqrt{2 \pi} \sigma)^{-} 1 \exp \left(-f^{2}\left(2 \sigma^{2}\right)^{1}\right) .
$$

Consider the transformation

$$
X^{2 k+1}(t)=f(t), k=0,1,2, \ldots,
$$

or

$$
x=f^{1 /(2 K+1)} .
$$


Then one gets

$$
\left\langle x^{2 n+2}\right\rangle=\int_{-\infty}^{+\infty} f^{\frac{2 n+2}{2 k+1}} W(f) \mathrm{d} f .
$$

Using Gamma Function

$$
\Gamma(\lambda)=\int_{0}^{+\infty} t^{\lambda-1} e^{-t} \mathrm{~d} t, \lambda>0
$$

one has

$$
\left\langle x^{2 n+2}\right\rangle=(\sqrt{2 \pi} \sigma)^{-1}\left(2 \sigma^{2}\right)^{\frac{1}{2}\left(1+\frac{2 n+2}{2 k+1}\right)} \Gamma\left(\frac{1}{2}+\frac{n+1}{2 k+1}\right) .
$$

In particular, $n=0$,

$$
\left\langle x^{2}\right\rangle=(\sqrt{2 \pi} \sigma)^{-1}\left(2 \sigma^{2}\right)^{\frac{2 k+3}{4 k+2}} \Gamma\left(\frac{2 k+3}{4 k+2}\right) .
$$

For the transformation

$$
x^{2 n+1}(t)=f(t)
$$

by setting $k=n$ in (5), (6) one gets

$$
\begin{aligned}
& \left\langle x^{2 n+2}\right\rangle=(\sqrt{2 \pi} \sigma)^{-1}\left(2 \sigma^{2}\right)^{\frac{4 n+3}{4 n+2}} \Gamma\left(\frac{4 n+3}{4 n+2}\right), \\
& \left\langle x^{2}\right\rangle=(\sqrt{2 \pi} \sigma)^{-1}\left(2 \sigma^{2}\right)^{\frac{2 n+3}{4 n+2}} \Gamma\left(\frac{2 n+3}{4 n+2}\right) .
\end{aligned}
$$

It follows

$$
\frac{\left\langle x^{2 n+2}\right\rangle}{\left\langle x^{2}\right\rangle^{n+1}}=\sqrt{\pi^{n}} \frac{\Gamma((4 n+3 /(4 n+2))}{\Gamma^{n+1}((2 n+3) /(4 n+2))} .
$$

\section{References}

1. Wu, W.F. and Lin, Y.K., 'Cumulant-neglect closure for nonlinear oscillator under random parametric and external excitations', Int. J. Nonlinear Mech., 19(4) (1984) 349-362.

2. Roberts, J.B. and Spanos, P.D., Random Vibration and Statistical Linearization, John Wiley \& Sons, New York, 1990.

3. Bolotin, V.V., Random Vibrations of Elastic Systems, Martinus Nijhoff, The Hague, 1984.

4. Ibrahim, R.A., Parametric Random Vibration, Research Studies Press, Taunton, UK, 1985.

5. Crandall, S.H., 'Non-Gaussian closure for random vibrations of nonlinear oscillators', Int. J. Nonlinear Mech., 15 (1980), 303-313.

6. Beaman, J.J. and Hedrick, J.K., 'Improved statistical linearization for analysis and control of nonlinear stochastic systems', J. Dyn. Syst. Meas. Control, ASME, 103 (1981) 14-21.

7. Caughey, T.K., 'On the response of nonlinear oscillator to stochastic excitation', Prob. Eng. Mech., 1 (1986) $2-4$.

8. Cai, G.Q. and Lin, Y.K., 'A new approximate solution technique for random excited nonlinear oscillators', Int. J. Nonlinear Mech., 23 (1988) 409-420.

9. Atalik, T.S, and Utku, S., 'Stochastic linearization of multi-degree-of-freedom nonlinear systems', J. Earth. Eng. Struct. Dynamics, 4 (1976) 411-420.

10. Noori, $\mathrm{M}$. and Davoodi, H., 'Comparison between equivalent linearization and Gaussian closure for random vibration analysis of several nonlinear systems', Int. J. Eng. Sci., 28 (9) (1990) 897-905.

11. Anh, N.D., Krause, R. and Schiehlen, W., 'Non-Gaussianity of the response of a Duffing oscillator to random excitation', to appear.

12. Ochi, M., 'Non-Gaussian random processes in ocean engineering', Prob. Eng. Mech., 1 (1986) 28-39.

13. Iyengar, R.N., 'Higher order linearization in nonlinear random vibration', Int. J. Nonlinear Mech., 23 (1988) 385-391. 